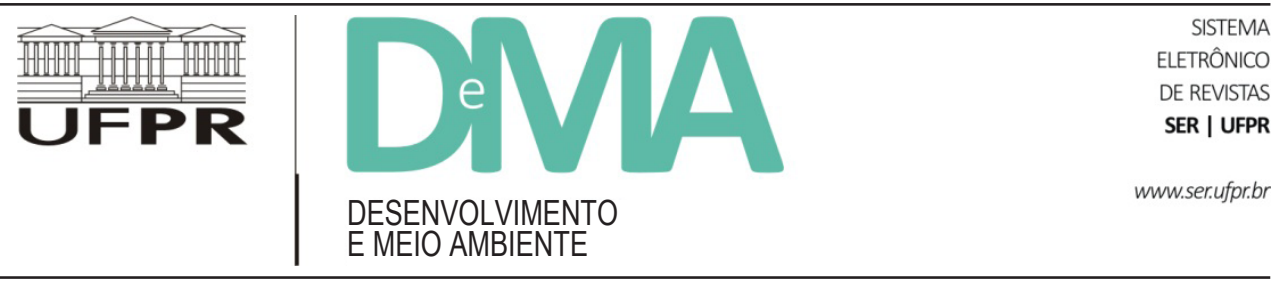

\title{
Diagnóstico e avaliação da eficiência da preservação do ambiente em Mato Grosso do Sul a partir da inclusão do pagamento de serviços ambientais
}

\section{Diagnosis and Assessment of Environmental Preservation's Efficiency in the State of Mato Grosso do Sul after the Inclusion of Payment for Environmental Services}

\author{
Jorge de Souza PINTO ${ }^{1,2^{*}}$, Daniel Massen FRAINER ${ }^{1,3}$, Ademir Kleber Morbeck de OLIVEIRA ${ }^{1}$, Celso Correia \\ de SOUZA $^{1}$ \\ ${ }^{1}$ Programa de Pós-Graduação em Meio Ambiente e Desenvolvimento Regional, Universidade Anhanguera-Uniderp, Campo Grande, MS, Brasil. \\ ${ }^{2}$ Universidade Federal de Mato Grosso do Sul, Campus do Pantanal (UFMS), Corumbá, MS, Brasil. \\ ${ }^{3}$ Universidade Estadual de Mato Grosso do Sul (UEMS), Dourados, MS, Brasil. \\ *E-mail de contato: jorge.pinto@ufms.br
}

Artigo recebido em 8 de maio de 2015, versão final aceita em 19 de novembro de 2015.

RESUMO: Este estudo como objetivo reunir e analisar informações sobre a conformidade de políticas de identificação, manutenção, conservação e preservação do ambiente e dos recursos naturais do Estado do Mato Grosso do Sul, como também avaliar a reciprocidade entre Pagamento de Serviços Ambientais do ICMS Ecológico e a preservação ambiental nas áreas territoriais das UCs nos municípios, com base na análise das informações do Sistema de Unidades de Conservação do Instituto de Meio Ambiente do Mato Grosso do Sul, no período de 2002 a 2014. Utilizou-se a Análise Envoltória de Dados nos dados obtidos das unidades de conservação de cada município, possibilitando posicionar os escores de eficiência do estado satisfatório de conservação e traçar o perfil do novo paradigma relativo a ações municipais frente à implantação, à manutenção, ao reconhecimento e à evolução de novas unidades no Estado. A importância dos valores repassados do ICMS Ecológico se reflete no empenho dos municípios em relação à criação de UCs, posto que, em 2002, havia 111 UCs em 52 municípios, perfazendo um repasse de R \$14,853 milhões. Já em 2014, eram 169 UCs em 66 municípios, com repasse total de $\mathrm{R} \$ 68,080$ milhões. Mostra-se no estudo a incipiência de ações proativas na gestão e no incremento de políticas de conservação do ambiente e que em poucos municípios ocorreram casos de eficiências localizadas em alguns anos, que não se mantiveram em períodos subsequentes, ressaltando a carência de estratégias duráveis para preservação dos recursos naturais no Estado.

Palavras-chave: sustentabilidade; gestão ambiental; custos ambientais; pagamento de serviços ambientais; análise envoltória de dados (DEA). 
ABSTRACT: This study aims at gathering and analyzing information on the compliance of policies of identification, maintenance, conservation and preservation of the environment and natural resources of the State, and also at assessing reciprocity between Environmental Services Payment (ESP) and the ecological environmental preservation in territorial of protected areas in the municipalities, based on the analysis of information from the Protected Areas' System from the Mato Grosso do Sul's Environmental Institute (IMASUL) from 2002 to 2014. The data enrollment analysis (DEA) was applied over the data obtained from protected areas of each municipality, enabling the positioning of the satisfactory efficiency conditions of conservation scores and profiling the new paradigm for the municipal actions against deployment, maintenance, recognition and evolution of new units in Mato Grosso do Sul. The importance of the values passed on to the Ecological ICMS reflects itself on the commitment of the municipalities in relation to the establishment of Protected Areas (PAs). Once in 2002, there were 111 protected areas in 52 municipalities of the state, adding up a transfer of $\$ 14,853,000$; then in 2014, there were 169 protected areas in 66 municipalities and a total wire of $\$ 68,080,000$. The application of data enrollment analysis (DEA) on social, economic and environmental indicators of each municipality data enabled protected areas to place efficient scores in satisfactory conditions in these municipalities in the state of MS, and profiling a new paradigm on municipal actions against deployment, maintenance and recognition of PAs. This study shows the inefficiency of proactive actions over management and development of environmental conservation policies, and that in a few cases there was some level of efficiency concentrated in certain years, which did not remain in subsequent periods, reinforcing the lack of long term strategies for conservation of natural resources in the state.

Keywords: sustainability; environmental conservation; environmental management; environmental costs; payment for environmental services.

\section{Introdução}

Em nível estadual e municipal, um dos instrumentos que tem colaborado na perspectiva de uma atenção diferenciada em relação às Unidades de Conservação (UCs) é o ICMS Ecológico que, com repasses financeiros e monitoramento das áreas dessas UCs, possibilita iniciativas de preservação e conservação dos recursos naturais e do ambiente no Brasil.

O repasse do ICMS Ecológico aos municípios surgiu da possibilidade dos Estados criarem critérios legais para a destinação de até $1 / 4$ dos $25 \%$ dos valores do Imposto sobre Operações Relativas à Circulação de Mercadorias e sobre Prestações de Serviços de Transporte Interestadual e Intermunicipal e de Comunicação (ICMS) arrecadados.

Assim, até 6,25\% do ICMS arrecadado nas unidades da federação pode tornar-se ICMS Ecológico e ser utilizado como ferramenta de incentivo à proteção da natureza e manutenção da biodiversidade; em MS é estabelecido em $5 \%$ do repasse, conforme tratam os estudos de Loureiro (2002), Ring (2008), Monte \& Silva (2009), Nohara et al. (2011), Rossi et al. (2011), Neves (2012) e Santos et al. (2012), que salientam a importância e a oportunidade do
ICMS Ecológico na transformação do cenário de investimentos do Estado na preservação ambiental.

O primeiro Estado brasileiro a adotar o ICMS Ecológico foi o Paraná, em 1991, seguido por São Paulo (1993), Minas Gerais (1995), Amapá (1996), Rio Grande do Sul (1997), Mato Grosso (2000), Mato Grosso do Sul (2000), Pernambuco (2000), Tocantins (2002), Rondônia (2005), Goiás (2007), Rio de Janeiro (2007), Ceará (2008), Piauí (2008), Pará (2009), Acre (2009) e Paraíba (2011).

Em dez estados sua adoção ainda se encontra em tramitação ou ainda não se propôs deliberação, caso dos Estados do Amazonas, Roraima, Alagoas, Bahia, Espírito Santo, Sergipe, Rio Grande do Norte, Maranhão, Distrito Federal e Santa Catarina, segundo dados da organização The Nature Conservancy (2014).

No ICMS Ecológico do Estado de Mato Grosso do Sul, existem dois critérios de avaliação dos municípios para credenciar seu recebimento: a adoção e a existência de ações públicas efetivas de tratamento de resíduos sólidos (lixo) no município, e o estado satisfatório das UCs na jurisdição do município, a partir dos quais o Estado retribui, com recursos financeiros, a título de Pagamento por Serviços Ambientais (PSA) de ICMS Ecológico, para 
que estes municípios continuem investindo em políticas públicas de conservação e preservação ambiental em UCs.

Assim como se discute a atuação do ICMS Ecológico como política pública de PSA, vários estudos internacionais caracterizam como essencial a necessidade de se criar e instaurar mecanismos transnacionais e internos, relacionados ao reconhecimento e à retribuição aos serviços ambientais nos países, como nos estudos de Kosoy et al. (2008), Maler et al. (2008), Ring (2008), Petheram \& Campbell (2010), Farley et al. (2010), Kemkes et al. (2010), Bettencourt \& Kaur (2011) e O'Farrell et al. (2011).

Diante da importância do aumento de preservação de recursos naturais e das UCs, tem-se ampliado a discussão de contrapartidas para a preservação destas unidades pela introdução de Políticas Públicas Ambientais, as quais consistem no conjunto de metas e instrumentos que procurem diminuir os impactos negativos da ação do homem sobre o ambiente.

Fundamentada na teoria econômica, a política ambiental toma como base as externalidades para o ambiente, os custos sociais e privados a fim de mensurar e instituir os mecanismos que sirvam como base para os gestores em suas decisões (Frey, 2000; May, 2010).

Por muito tempo, o ambiente não se fez presente nas decisões dos gestores e com a omissão, no processo de industrialização brasileira, de cuidados com os recursos naturais e com a política ambiental, esta não fazendo parte do processo, tornam-se latentes os exemplos de descaso com os aspectos ambientais no Brasil, como as indústrias, que apresentam um grau elevado de poluição, e até mesmo a própria agropecuária e suas fronteiras agrícolas, que têm comprometido recursos naturais e causado impactos ambientais (Lustosa et al., 2003).

Portanto, o modelo da política ambiental brasileira teve como base o controle da poluição e a criação de unidades de conservação da natureza. Ficaram excluídos desse modelo o crescimento populacional e o saneamento básico, que são impactantes nas políticas em relação ao meio ambiente, sendo objetos de política própria, não vinculados com a questão ambiental (Nascimento et al., 2013).

Observando a necessária evolução de instrumentos de política pública ambiental, com desdobramentos tanto públicos quanto privados, tem-se a síntese de que estes instrumentos foram criados para internalizar o custo externo ambiental, podendo-se classificá-los como:
Instrumentos de Comando-e-Controle: controle ou proibição de produto; controle de processo; proibição ou restrição de atividades; controle do uso de recursos naturais; padrão de poluição para fontes específicas;

$\checkmark$ Instrumentos de Comunicação: fornecimento de informação; acordos; criação de redes; sistema de gestão ambiental; selos ambientais; marketing ambiental.

$\checkmark$ Instrumentos Econômicos: taxas e tarifas; subsídios; certificados de emissão transacionáveis; sistemas de devolução de depósitos; impostos (ICMS-Ecológico) (Lustosa \& Young, 2002).

Assim, verifica-se que as políticas ambientais estão baseadas quase que exclusivamente nos instrumentos de comando-e-controle, que envolvem restrições gerenciais e quantitativas ao uso dos bens e serviços ambientais e aos instrumentos econômicos, sendo que esses incentivos atuam na forma de preços e na forma de prêmios (Nascimento et al., 2013).

Vários autores sugerem a utilização conjunta do instrumento de comando-e-controle e do econômico, pois o instrumento de comando-e-controle, por tratar o poluidor como provedor de delinquências ambientais e por ter apresentado resultados não satisfatórios na obtenção de objetivos ambientais, necessita ser complementado pelos instrumentos econômicos para alcançar resultados mais adequados e efetivos em relação à preservação do ambiente (Nascimento et al., 2013).

A introdução dos PSA na rotina de mecanismos de sustentabilidade tem assumido o arcabouço de permitir que os recursos possam vir a ser aplicados em resposta aos desafios de preservar o ambiente, gerando mais resultados econômicos, com menor dispêndio de recursos naturais (Pagiola et al., 2005).

Em países como Costa Rica, Colômbia, México e Equador, já se tem obtido bons resultados com a introdução do PSA e o reconhecimento dos serviços ambientais do uso da terra e dos recursos hídricos, permitindo a estes países investir em programas que favoreçam uma coletividade maior com a manutenção da preservação do ambiente e dos recursos naturais (Kosmus \& Cordero, 2009). 
Portanto, este estudo teve como objetivo reunir e analisar informações sobre a conformidade de políticas de identificação, manutenção, conservação e preservação do ambiente e dos recursos naturais de Mato Grosso do Sul, como também avaliar a reciprocidade entre Pagamento de Serviços Ambientais do ICMS Ecológico e a preservação ambiental nas áreas territoriais das Unidades de Conservação (UCs) nos municípios, com base na análise das informações do Sistema de Unidades de Conservação do Instituto de Meio Ambiente do Mato Grosso do Sul, no período de 2002 a 2014.

\section{Material e métodos}

Considerando que cada região do Estado de Mato Grosso do Sul (MS) possui características específicas em relação à atividade econômica predominante e, consequentemente, repercussão específica na existência e consolidação de UCs, as informações das UCs foram segregadas por região e seus respectivos municípios, conforme divisão oficial descrita no Caderno Geoambiental das Regiões de Planejamento do MS, da Secretaria de Planejamento e Coordenação de Mato Grosso do Sul (SEPLAN-MS).

O Estado possui oito regiões: Norte (10 municípios), Sul (15), Leste (8), Cone Sul (10), Bolsão (10), Pantanal (5), Sudoeste (8) e Região da Grande Dourados (12), divididas, ainda, em duas bacias hidrográficas: Bacia do Rio Paraguai (BH-RPY) e Bacia do Rio Paraná (BH-RPR) (Figura 1).

O levantamento de dados sobre a avaliação e a classificação das UCs no Estado de Mato Grosso do Sul teve por base os documentos oficiais publicados pela

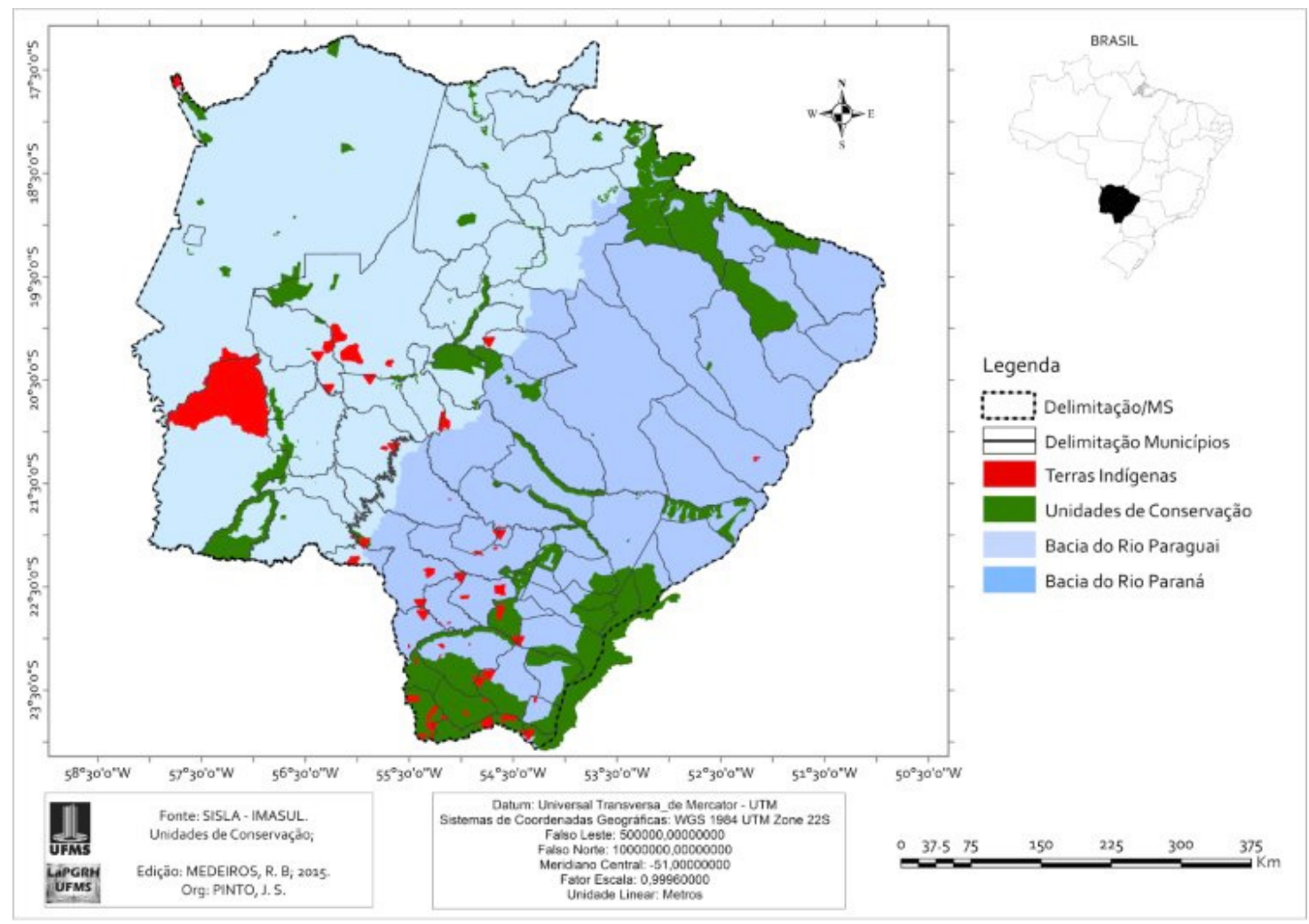

FIGURA 1 - Área de distribuição das Unidades de Conservação no Estado de Mato Grosso do Sul, Brasil, em 2014.

FONTE: Sistema Interativo de Suporte ao Licenciamento Ambiental - SISLA/IMASUL (2014). 
Secretaria Estadual de Meio Ambiente e Desenvolvimento Econômico de Mato Grosso do Sul (SEMAC/ MS), gerados pelo Instituto de Meio Ambiente de Mato Grosso do Sul (IMASUL) e constantes do banco de dados da gerência de cada UC, para monitoramento das áreas cadastradas que se encontram em conformidade para repasses dos recursos do ICMS Ecológico aos municípios.

Em relação às UCs, foram levantadas informações sobre sua designação, a área total em (ha) e a área que é considerada satisfatória (conformidade de preservação), disciplinada pelo Decreto 10.478/2001, que regulamenta a qualificação das áreas de conservação para aplicação do ICMS Ecológico e, portanto, para os fins de credenciamento ao ICMS Ecológico, assim como os indicadores de avaliação e classificação de recebimento dos recursos aos municípios (Mato Grosso do Sul, 2001).
Aplicou-se neste estudo o método de Análise Envoltória de Dados (Data Envelopment Analysis DEA), para determinação da UCs eficientes, conforme Angulo-Meza et al. (2007) e Senra et al. (2007), mais especificamente o DEA-BCC orientado ao produto, sendo utilizado com dois insumos e um produto. Como insumos: 1) os repasses de ICMS Ecológico em reciprocidade à manutenção e à preservação de UC em seus domínios municipais, e 2) o índice ambiental calculado para cada um dos municípios que possui UC no cadastro do IMASUL, que é feito anualmente e permite avaliar a conformidade e o estado satisfatório das unidades e suas especificidades de manejo (Figura 2).

Ainda, com base nos dados das UCs, foram compilados os percentuais em hectares do território de cada

\section{METOD OLOGIA DE CAL CULO DO INDICE AMBIENTAL (IA) - ICMS ECOLOGICO/MS}

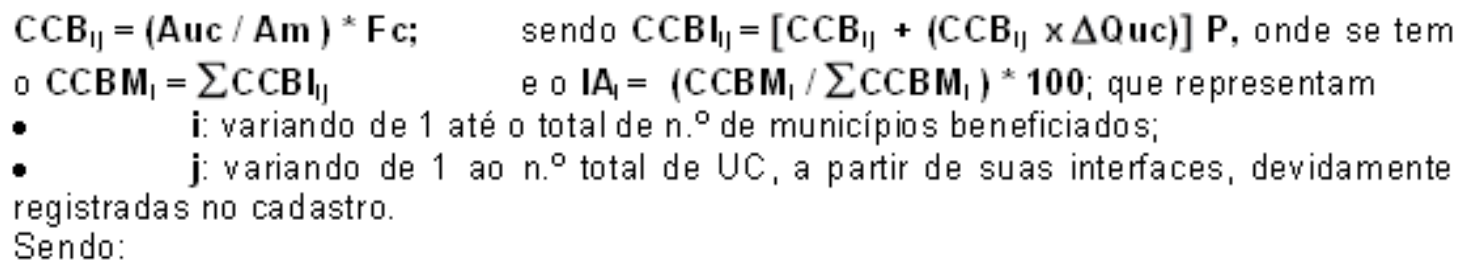

- $\mathrm{CCB}_{\mathrm{I}}$ : Coeficiente de Conservaçẫo da Biodiversidade básico;

- Auc: porçẫo da área da unidade de conservaçẫo ou outras áreas especialmente protegidas criadas na forma da lei, contida no município, distribuída de acordo com sua qualidade física e procedimentos subsequentes;

- Am: área total do território municipal fornecido por órgẫo oficial;

- Fc: fator de conservaçã̃o, parâmetro variável, atribuído às unidades de conservaçẵo e outros espaços especialmente protegidos em funçẫo das respectivas categorias de manejo;

- CCBI I: Coeficiente de Conservaçẫo da Biodiversidade por Interface;

- $\Delta Q u c:$ variação da qualidade da UC ou outro espaço especialmente protegido;

- P: peso ponderado;

- CCBM : Coeficiente de Conservaçẫo da Biodiversidade para o Município, equivalente a soma de todos os Coeficientes de Conservaçẫo de Interface calculados para um determinado município;

- $\mathrm{IA}_{1}$ : índice percentual calculado, a ser destinado ao município, denominado indice ambiental.

FIGURA 2 - Metodologia de cálculo do Índice Ambiental das Unidades de Conservação no Estado de Mato Grosso do Sul, Brasil. FONTE: Mato Grosso do Sul, Decreto n. 10.478 de 31/08/2001. 
município que compõe suas UCs, bem como os repasses de ICMS Ecológico ocorridos no período de 2002 a 2014.

O DEA fornece dois tipos de modelos para análise. Charnes et al., (1978) elaboraram o modelo CCR, também conhecido por Constant Returns to Scale (CRS), o qual avalia a eficiência total, determinando as Decision Making Units (DMU's) eficientes da amostra, além de fornecer informação sobre as distâncias das DMU's ineficientes da fronteira de eficiência.

Banker et al., (1984) elaboraram o modelo conhecido como BCC, ou Variable Returns to Scale (VRS). Este modelo, conforme os autores, “[...] permite a projeção de cada DMU ineficiente sobre a superfície de fronteira (envoltória) determinada pelas DMU's eficientes de tamanho compatível" (Soares de Mello et al., 2005; 2006).

Para realizar a análise através de um desses dois modelos, existe a necessidade de fixação da ótica de análise, ou seja, se a análise será orientada pelos insumos ou pelos produtos. A análise orientada pelos produtos mantém fixa a quantidade insumos e maximiza as quantidades de outputs. Já o modelo orientado pelos insumos minimiza as quantidades de inputs, mantendo fixas as quantidades de outputs (Angulo-Meza et al., 2007; Senra et al., 2007). Na aplicação do DEA neste estudo, foi utilizado o modelo BCC com orientação voltada para a maximização do output.

Devido às características matemáticas do modelo BCC, as DMU's que têm o menor valor de um dos insumos ou o maior valor de um dos produtos são consideradas eficientes, mesmo que as relações com as demais variáveis não sejam as melhores (Angulo-Meza \& Lins, 2002).

A normalização adotada neste estudo utiliza o método descrito por Angulo-Meza et al. (2005), que não considera os valores máximos e mínimos efetivamente atingidos para a eficiência e o número de DMU's na fronteira para cada quantidade de variáveis, mas sim os valores extremos teoricamente alcançáveis, em que o número de DMU's na fronteira está no conjunto $\{1,2,3, \ldots, n\}$, sendo que " $n$ " é o número total de DMU's e a eficiência média também vai estar classificada no intervalo $[0,1]$. Portanto, para obter a normalização das eficiências médias para cada conjunto de variáveis, levou-se em conta que quanto maior a eficiência média, maior deve ser o valor dessa variável (Angulo-Meza et al., 2007).

Já para obter a normalização da quantidade de DMU's eficientes para cada conjunto de variáveis, considera-se que quanto maior o número de DMU's na fronteira menor é o valor da variável (Soares de Mello et al., 2005).

A aplicação da normalização foi usada em cada etapa do método para escolher uma próxima variável a ser inserida ou que irá compor o modelo de análise. No caso do número de variáveis não ser grande, pode ser calculada para todas as combinações possíveis de inputs e outputs, permitindo que o conjunto final de variáveis a ser escolhido seja aquele que apresentar o maior índice (Angulo-Meza et al., 2005; Soares de Mello et al., 2006; Senra et al., 2007).

Conforme descrito no modelo DEA adotado neste estudo, foram utilizados os critérios estabelecidos a partir dos quais se podem obter os resultados do comportamento e estado satisfatório das UCs em MS, no período de 2002 a 2014.

\section{Resultados e discussões}

Em Mato Grosso do Sul, a partir da introdução do ICMS Ecológico, com a avaliação das áreas em 2001 para início de repasses em 2002, já se iniciou um movimento de criação de novas UCs; em 2002, ocorreu um aumento gradual de hectares incorporados recebendo recursos.

O crescimento, em termos de área, foi de mais de 300\% em áreas consideradas em conformidade até 2014, indicando a aceitação, pelos municípios, deste tipo de incentivo.

Em 2002, o Estado possuía nove modalidades de UC, sendo 14 APA’s (Áreas de Proteção Ambiental), 20 RPPN's (Reservas Particulares do Patrimônio Natural), 44 TI's (Terras Indígenas), 1 MN (Monumento Natural), 4 RC's (Rios Cênicos), 11 PE's (Parques Estaduais), 4 PN's (Parques Nacionais), 3 EP's (Estradas Parques) e 10 PNM's (Parques Naturais Municipais). Já em 2014 passa a ter 52 APA's, 43 RPPN's, 36 TI's, 7 MN's, 10 PE's, 9 PN's e 12 PNM's, consignando o engajamento 
dos municípios com os PSA dos repasses do ICMS Ecológico, com destaque para RPPN's e APA's.

A distribuição do ICMS Ecológico para os municípios com UCs obedece a um conjunto estruturado de normas e, nos casos em que a UC é considerada em estado satisfatório de conservação, a mesma figurará integralmente para fins de partição dos recursos. Em casos extremos, onde a UC é avaliada em não conformidade de preservação pelo IMASUL, a mesma é excluída do cadastro estadual, podendo retornar, desde que restabeleça bases satisfatórias de conservação.

Segundo dados obtidos na gerência de UC do IMASUL, até 2014 são 12 Áreas de Proteção Ambiental (APA) que possuem planos de manejo (PM) e 8 em elaboração; Reserva Particular do Patrimônio Natural (RPPN), 14 com PM; PE's, 5 com PM e os Parques Naturais Municipais (PNM), 7 com PM e um PM em elaboração; Parques Nacionais (PN), 3 com PM, além de dois Monumentos Naturais (MN) com Plano de Manejo. É significativo o volume de áreas e seu respectivo crescimento, embora a adoção e a implantação de planos de manejo ainda sejam incipientes em muitas unidades.

Em relação à totalidade das UCs, em 2014 apenas 43 possuíam PM, o que permitiu a análise e a conciliação com o manejo e a preservação existente e, assim, a manutenção da conformidade para figurar como UC preservada na avaliação técnica do IMASUL.

De acordo com Loureiro (2002), o ICMS Ecológico evoluiu, transformando-se ao longo do tempo também em instrumento de incentivo, direto e indireto, à conservação ambiental, sendo hoje o que mais o caracteriza em detrimento de sua gênese de compensação.

$\mathrm{Na}$ Tabela 1, considerando a jurisdição de UCs, se observam em MS os efeitos da adoção do PSA do ICMS Ecológico, de 2002 até 2014, onde mostra em evolução a criação e a manutenção de UCs, sendo observado ainda o crescimento na adesão de novas iniciativas de criação e de manutenção de novas áreas de UCs, que permitem prospectar um cenário favorável à conservação e à preservação do ambiente no Estado.

Os valores de ICMS Ecológico repassado aos municípios, em sua essência, funcionaram como uma ferramenta de incentivo à proteção e à manutenção da natureza e da biodiversidade.

Os dados da Tabela 1 mostram que, com exceção das áreas federais, que sofreram uma redução de $21 \%$, todos os tipos de unidades aumentaram suas áreas, com destaque para as UCs estaduais, com aumento de mais de $500 \%$, seguidas pelas UCs municipais, $+60 \%$, e UCs privadas, $+26 \%$. No total, ocorreu um aumento de UCs de + de $200 \%$.

A redução nas UCs federais, Tabela 1, está relacionada à avaliação da conformidade de conservação do ambiente na área, realizada pelo IMASUL anualmente, tendo como caso mais emblemático a redução de conformidade na TI Kadiwéu em Porto Murtinho de $538.535,78$ hectares em 2002 para $376.591,78$ hectares em 2014, sendo que na jurisdição federal, neste intervalo de tempo, também houve ingresso/supressão de outras áreas de UCs, em razão do acompanhamento em relação à preservação do ambiente.

Em alguns casos, a área integral da UC não é considerada, por não reunir as condições mínimas exigidas de preservação, o que leva a diferenças significativas

TABELA 1 - Jurisdição de gestão e evolução das áreas de Unidades de Conservação (UCs) no Estado de Mato Grosso do $\mathrm{Sul}-2002$ a 2014.

\begin{tabular}{|c|c|c|c|}
\hline UCs & $\begin{array}{c}\text { Área } \\
\text { protegida } \\
\text { (ha) }\end{array}$ & $\begin{array}{c}\% \text { da } \\
\text { área total } \\
\text { protegida } \\
\end{array}$ & $\begin{array}{c}\text { Área protegida em } \\
\text { \% da área total } \\
\text { de MS }\end{array}$ \\
\hline \multicolumn{4}{|l|}{ Privada } \\
\hline 2002 & $104.270,1$ & $6 \%$ & $0,29 \%$ \\
\hline 2014 & $132.151,7$ & $3 \%$ & $0,37 \%$ \\
\hline \multicolumn{4}{|l|}{ Municipal } \\
\hline 2002 & $115.076,0$ & $7 \%$ & $0,32 \%$ \\
\hline 2014 & $184.150,7$ & $4 \%$ & $0,52 \%$ \\
\hline \multicolumn{4}{|l|}{ Estadual } \\
\hline 2002 & $755.428,6$ & $45 \%$ & $2,12 \%$ \\
\hline 2014 & $4.327 .633,8$ & $83 \%$ & $12,12 \%$ \\
\hline \multicolumn{4}{|l|}{ Federal } \\
\hline 2002 & $710.037,0$ & $42 \%$ & $1,99 \%$ \\
\hline 2014 & $559.942,8$ & $11 \%$ & $1,57 \%$ \\
\hline \multicolumn{4}{|l|}{ Total } \\
\hline 2002 & $1.684 .811,7$ & $100 \%$ & $4,72 \%$ \\
\hline 2014 & $5.203 .878,9$ & $100 \%$ & $14,57 \%$ \\
\hline
\end{tabular}

FONTE: Dados da pesquisa. 
nas áreas consideradas não satisfatórias. Por exemplo, em 2006, 438.603,3 hectares e, em 2014, 480.748,9 hectares foram considerados não satisfatórios (Tabela 2), indicando problemas de gerenciamento em algumas unidades de conservação.

Em 2006, o problema ocorreu no município de Aquidauana, em que foram inseridas e avaliadas três UCs, identificadas como APA do Rio Aquidauana, 477.255,9 ha, APA do Rio Negro, 241.951,2 ha e APA do Taboco, 143.820,0 ha.

Entretanto, por não reunirem perspectivas de continuidade e conformidade com as normas de avaliação técnica do IMASUL, a partir de 2007, não constaram das áreas em conformidade do município para as UCs, ocasionando variações nos hectares das UCs (Tabela 2). As quantidades de áreas avaliadas e consideradas conformes no estudo são obtidas em dados do IMASUL, sendo que em 2003 e 2004, assim como 2011 e 2012, as áreas apresentam os mesmos montantes.

TABELA 2 - Área total, satisfatória e diferença, em hectares, das Unidades de Conservação (UCs) no Estado de Mato Grosso do Sul, de 2002 a 2014.

\begin{tabular}{cccc}
\hline Ano & $\begin{array}{c}\text { Área total das } \\
\text { UCs }\end{array}$ & $\begin{array}{c}\text { Área satisfatória } \\
\text { das UCs }\end{array}$ & Diferença \\
\hline 2002 & $1.628 .046,5$ & $1.628 .028,0$ & 18,50 \\
2003 & $1.715 .922,8$ & $1.715 .902,3$ & 20,50 \\
2004 & $1.715 .922,8$ & $1.715 .902,3$ & 20,50 \\
2005 & $2.568 .471,3$ & $2.541 .764,2$ & $26.707,1$ \\
2006 & $4.664 .189,5$ & $4.225 .586,2$ & $438.603,3$ \\
2007 & $3.961 .880,3$ & $3.571 .224,5$ & $390.655,8$ \\
2008 & $3.876 .221,6$ & $3.527 .173,1$ & $349.048,4$ \\
2009 & $4.216 .083,8$ & $3.935 .109,0$ & $280.974,7$ \\
2010 & $4.977 .899,5$ & $4.830 .343,9$ & $147.555,6$ \\
2011 & $5.532 .084,9$ & $5.244 .513,9$ & $287.571,0$ \\
2012 & $5.532 .084,9$ & $5.244 .513,9$ & $287.571,0$ \\
2013 & $5.550 .564,9$ & $5.242 .927,3$ & $307.637,6$ \\
2014 & $5.684 .627,8$ & $5.203 .878,9$ & $480.748,9$ \\
\hline
\end{tabular}

FONTE: Dados da pesquisa.

No desenvolvimento evolutivo de criação de uma UC em Mato Grosso do Sul, dois tipos de unidades se destacaram, as APA's e as RPPN's, preconizando a estrutura de conservação desde 2002. A primeira, a APA, é um tipo de UC em que não é necessário o processo de desapropriação da área, permanecendo os proprietários no local, com suas atividades tradicionais; já na segunda, a RPPN, o proprietário cria a unidade em um local de sua propriedade, normalmente em pontos em que a atividade agropecuária é mais difícil ou que apresenta potencial para o turismo ambiental.

Em 2002, no início do processo de implantação das UCs, a área cadastrada satisfatória era de 1.628.028,0 hectares, com 111 UCs em 49 municípios, representando $5,8 \%$ da área territorial total dos municípios até então cadastrados. Já em 2007, passou para 3.571.224,5 hectares, com 142 UCs em 56 municípios, representando $12,6 \%$ da área territorial total dos municípios.

Em 2014, a área passou para 5.203.878,9 hectares, contando com 67 municípios e um total de 169 UCs, representando $16,3 \%$ da área territorial total, demonstrando uma evolução em relação à criação e à manutenção de UC e indicando uma melhor abordagem por parte dos municípios em relação às oportunidades de se ter em sua circunscrição áreas de interesse em conservação e preservação ambiental e, com isso, carreando mais recursos para aperfeiçoar sua gestão ambiental (Tabela 3).

TABELA 3 - Áreas territoriais satisfatórias das Unidades de Conservação (UCs) em MS e relação percentual com as áreas dos municípios e evolução dos repasses de PSA do ICMS Ecológico do Estado de Mato Grosso do Sul, Brasil, de 2002 a 2014.

\begin{tabular}{cccc}
\hline Ano & $\begin{array}{c}\text { Hectares de UC } \\
\text { satisfatórias }\end{array}$ & $\begin{array}{c}\text { \% área das } \\
\text { UCs com a dos } \\
\text { municípios }\end{array}$ & $\begin{array}{c}\text { Valor PSA - } \\
\text { ICMS Ecológico }\end{array}$ \\
\hline 2002 & $1.628 .028,0$ & 5,8 & $14.853 .031,40$ \\
2003 & $1.715 .902,3$ & 6,2 & $14.853 .031,40$ \\
2004 & $1.715 .902,3$ & 6,2 & $26.722 .525,09$ \\
2005 & $2.541 .764,2$ & 9,8 & $30.741 .895,35$ \\
2006 & $4.225 .586,2$ & 14,9 & $36.314 .371,83$ \\
2007 & $3.571 .224,5$ & 12,6 & $35.392 .974,28$ \\
2008 & $3.527 .173,1$ & 12,4 & $41.786 .005,67$ \\
2009 & $3.935 .109,0$ & 13,3 & $39.470 .197,10$ \\
2010 & $4.830 .343,9$ & 15,6 & $43.578 .020,16$ \\
2011 & $5.244 .513,9$ & 17,7 & $51.216 .180,08$ \\
2012 & $5.244 .513,9$ & 16,6 & $55.159 .198,12$ \\
2013 & $5.242 .927,3$ & 16,6 & $62.566 .357,22$ \\
2014 & $5.203 .878,9$ & 16,3 & $68.080 .425,25$ \\
\hline
\end{tabular}

FONTE: Dados da pesquisa. 
Em 2002, com 49 municípios cadastrados, foram repassados em PSA de ICMS Ecológico R\$ 14.853.031,40, atendendo a 111 UCs. Em 2014, com 67 municípios, tem-se o PSA no montante de R\$ $68.080 .425,25$, o que representa uma significativa evolução das políticas públicas estaduais e municipais em relação à preservação do ambiente (Tabela 3 ).

É importante observar que os valores de repasse de PSA de ICMS Ecológico podem ser muito significativos para determinados municípios, principalmente os pequenos, com poucos recursos financeiros, dependentes da transferência de valores do Estado, tais como Alcinópolis, Caracol, Coronel Sapucaia, Dois Irmãos do Buriti, Douradina, Eldorado, Japorã, Jateí, Paranhos, Sonora e Taquarussu.

Mesmo municípios que possuíam atividades econômicas mais consolidadas, tais como Antônio João, Aquidauana, Bonito, Chapadão do Sul, Corumbá, Fátima do Sul, Jardim, Mundo Novo, Sete Quedas e Terenos, receberam valores de PSA do ICMS Ecológico que são importantes para suas finanças, demonstrando os impactos destas transferências de recursos para a manutenção destes locais.

Em relação às regiões do Estado (Figura 3), nota-se que os comportamentos das tendências de crescimentos das áreas de preservações, inclusive com as criações e manutenções de UCs, são menores nas regiões onde se observa um predomínio das atividades agrícolas, com destaque para a produção de grãos, reflorestamento e produção de celulose, envolvendo grande parte das regiões Norte, Sul, Cone Sul, Leste, Grande Dourados e Região do Bolsão, onde as UCs ainda são incipientes frente à necessidade de postura proativa de conservação do ambiente.

Atualmente, restam pequenas parcelas de recursos naturais florestais nativos remanescentes, que hoje necessitam de políticas públicas de incentivo de preservação nos municípios, propiciando a possibilidade de manutenção destes locais, para mitigar a acentuada depleção do ambiente decorrente da intensa atividade antropogênica de exploração econômica dos recursos naturais.

Também merecem destaque as regiões Leste, do Pantanal e da Grande Dourados (Figura 3), que não apresentam evolução em termos de UCs, fato motivado pela expansão da cultura do eucalipto, expansão da pecuária e da lavoura, respectivamente, o que lhes concebe comportamentos mais convencionais em relação à agregação de áreas de UCs. Isto ocorre não que estas regiões sejam áreas potenciais a fazerem parte do rol de UC, mas pela capacidade ainda incipiente de mobilização em torno de

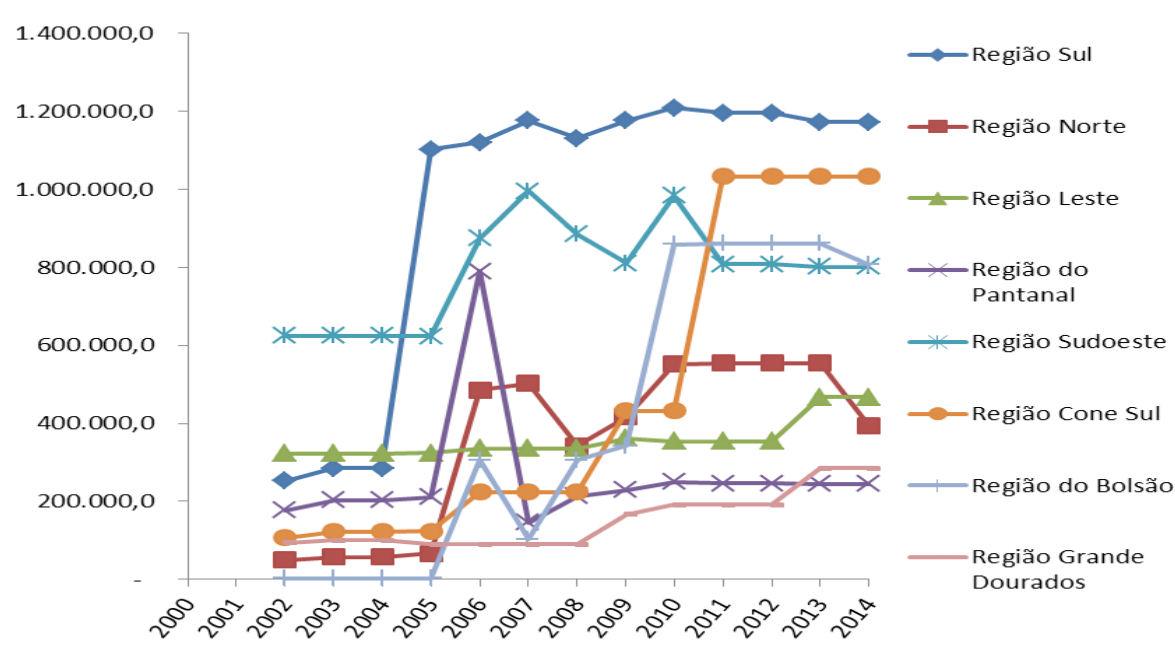

FIGURA 3 - Áreas de conservação, por região e em hectares, com repasse do ICMS Ecológico no período 2002/2014, Mato Grosso do Sul, Brasil. 
um desafio, que é conciliar a atividade econômica com a manutenção da preservação ambiental.

Em outras regiões, como a Cone Sul, a Sudoeste e a Sul, observa-se um comportamento ascendente em termos de composição de adesões de UCs (Figura 3), mesmo que sejam oriundas de mitigações de consequências de impactos ambientais significativos ocorridos, como construção de hidrelétricas, caso do Parque Estadual Várzeas do Rio Ivinhema, onde se tenta constituir um arcabouço que favoreça a mitigação e a recuperação de áreas que já sofreram muita depleção.

Também se deve levar em consideração a existência de um delimitador de bacia hidrográfica, a Serra de Maracajú, que corta o Estado no sentido Norte-Sul, dividindo-o em duas grandes e representativas bacias ambientais, a Bacia Hidrográfica do Rio Paraguai (BH-RPY), a qual possui 29 municípios com UCs, sendo que uma análise descritiva demonstra uma mediana das áreas preservadas de 1.081,1 hectares em 2002 e, em 2014, de 10.316,6 hectares em áreas satisfatórias. Na Figura 4, os índices de eficiências anuais das UCs dos 26 municípios da BH-RPY que possuíam UCs são apresentados.
Mesmo com extensas áreas territoriais, os $26 \mathrm{mu}-$ nicípios possuem falta de disciplinamento nos investimentos em relação à preservação dos recursos naturais, ou seja, os índices médios de eficiência obtidos pelos municípios, que destacam Alcinópolis $(0,4018)$, Aquidauana $(0,4708)$, Bodoquena $(0,4261)$, Bonito $(0,3865)$, Corumbá $(0,3534)$, Coxim $(0,6992)$, Jardim $(0,3701)$, Miranda $(0,4686)$ e Nioaque $(0,4740)$, importantes representantes do ambiente pantaneiro, demonstram uma situação em que suas maiores riquezas territoriais, que são o ambiente e sua biodiversidade, não estão tendo a devida atenção para sua conservação e preservação, como mostram os índices obtidos (Figura 4).

A apresentação da evolução dos índices de eficiência possibilita verificar a existência de um déficit em relação aos mecanismos de políticas públicas de incentivo à preservação e à conservação na região que abriga importante diversidade de recursos ambientais, como a própria planície do Pantanal.

Os resultados ainda apontam uma dispersão significativa dos índices, indicando a existência de déficits significativos em relação aos 23 municípios da BH-RPY,

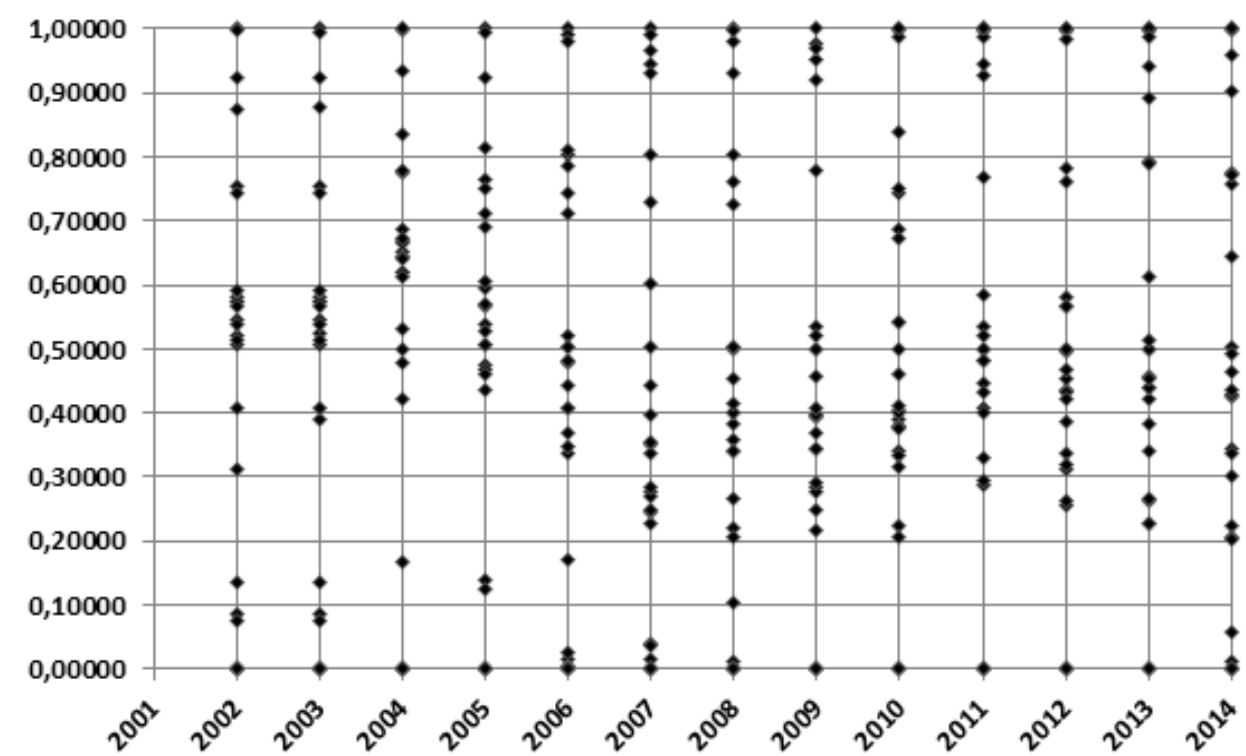

FIGURA 4 - Índices de eficiência anuais das Unidades de Conservação, por município do Estado de Mato Grosso do Sul, Bacia Hidrográfica do Paraguai, de 2002 a 2014, obtidos pela ferramenta DEA/BCC. 
prejudicando a almejada conciliação de recursos dos PSA e a preservação do ambiente (Figura 4).

A Bacia Hidrográfica do Rio Paraná (BH-RPR) possui 44 municípios com UCs e uma mediana das áreas preservadas de 77,1 hectares em 2002 e de 31.542,8 hectares em 2014 de áreas de UC satisfatórias (Figura 5).

Nos municípios com UCs são encontrados comportamentos deficitários em relação aos índices médios de eficiência obtidos nos 13 anos analisados, com destaque para Água Clara $(0,4073)$, Brasilândia $(0,2929)$, Campo Grande (0,7067), Douradina (0,1459), Dourados $(0,2506)$, Jatei $(0,2960)$, Maracajú $(0,2042)$, Naviraí $(0,5207)$ e Três Lagoas $(0,1334)$, o que demonstra também que nestas áreas parte da diversidade ambiental não está tendo a devida atenção para sua conservação e preservação (Figura 5).

Os escores de eficiência municipal para municípios da BH-RPY e BH-RPR (Tabelas 4 e 5) mostram que a maior parte dos municípios tem baixos índices de eficiência ambiental, ou seja, em um intervalo entre 0,0 e 1,0, a premissa assumida no estudo é de que os escores inferiores a 0,8 estão mais tendentes ao declínio de eficiência ambiental e os escores a partir de 0,8 até 1,0 apresentam ascendência de eficiência ambiental na conjugação do ICMS Ecológico em relação a hectares de UCs preservados nos municípios.

Em alguns períodos ocorreu o alcance de escores anuais de eficiência 1,0, mas que não se sustentaram ao longo dos anos, caracterizando deficiências de manejo em relação à preservação das UCs e do ambiente.

Verifica-se uma grande oscilação entre os escores de quantidade dos municípios que possuem UCs cadastradas, sendo que mais de $80 \%$ se encontram com índices de até 0,80 , níveis insustentáveis, e os outros $20 \%$ transitam entre 0,80 e 1,0, ao longo do período de 2002 a 2014, demonstrando pouca eficiência média geral na busca pelo desenvolvimento sustentável das UCs que abrigam (Tabelas 4 e 5).

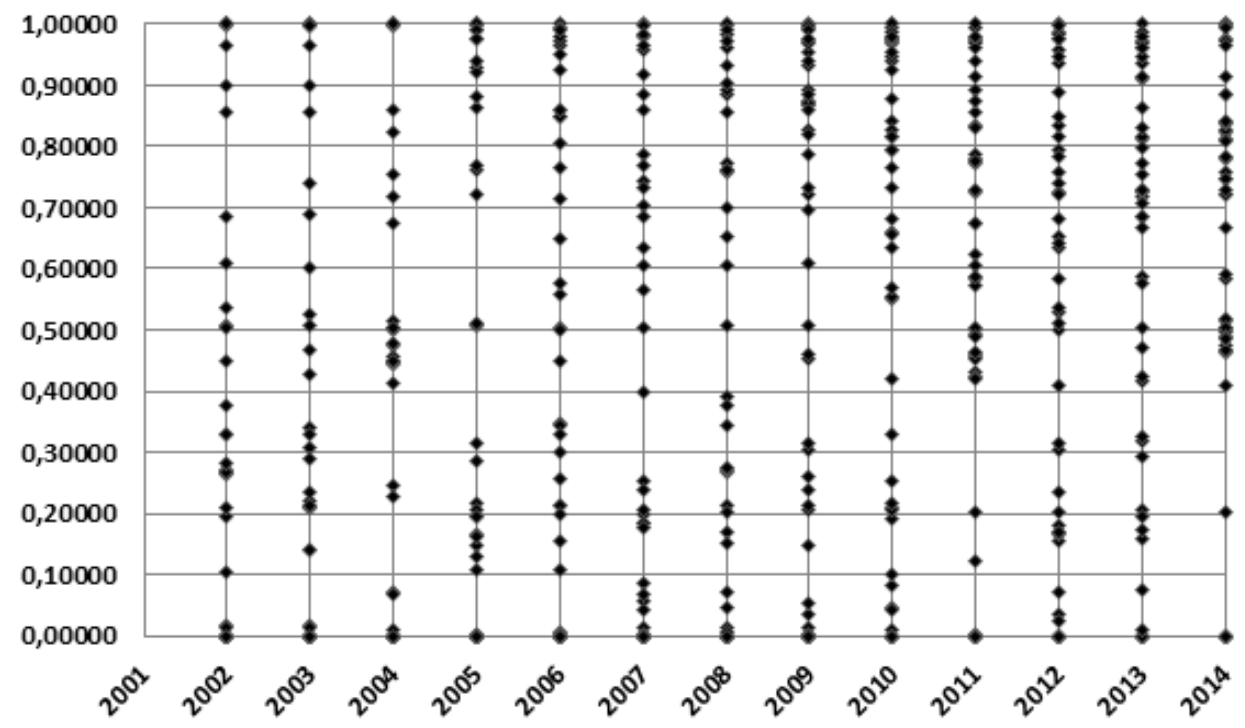

FIGURA 5 - Índices de eficiência anuais das Unidades de Conservação, por município do Estado de Mato Grosso do Sul da Bacia Hidrográfica do Paraná, de 2002 a 2014, obtidos pela ferramenta DEA/BCC. 
TABELA4 - Escores de eficiência municipal na Bacia Hidrografia do Rio Paraguai, quantidade de municípios e frequências percentuais desses municípios, de 2002 a 2014.

\begin{tabular}{|c|c|c|c|c|c|c|}
\hline $\begin{array}{c}\text { Escores de } \\
\text { eficiência }\end{array}$ & & $\begin{array}{l}0,1 \text { a } \\
<0,3 \\
\end{array}$ & $\begin{array}{l}0,3 \text { a } \\
<0,5 \\
\end{array}$ & $\begin{array}{l}0,5 \text { a } \\
<0,8 \\
\end{array}$ & $\begin{array}{l}0,8 \text { a } \\
<1,0 \\
\end{array}$ & 1,0 \\
\hline \multirow{2}{*}{2002} & QM & 3 & 2 & 6 & 3 & 1 \\
\hline & $\%$ & 14,29 & 9,52 & 28,57 & 14,29 & 4,76 \\
\hline \multirow{2}{*}{2004} & QM & 2 & 2 & 8 & 3 & 1 \\
\hline & $\%$ & 9,09 & 9,09 & 36,36 & 13,64 & 4,55 \\
\hline \multirow{2}{*}{2006} & QM & 4 & 8 & 5 & 4 & 1 \\
\hline & $\%$ & 17,39 & 34,78 & 21,74 & 17,39 & 4,35 \\
\hline \multirow{2}{*}{2008} & QM & 5 & 9 & 2 & 4 & 1 \\
\hline & $\%$ & 21,74 & 39,13 & 8,70 & 17,39 & 4,35 \\
\hline \multirow{2}{*}{2010} & QM & 3 & 11 & 3 & 3 & 1 \\
\hline & $\%$ & 12,00 & 44,00 & 12,00 & 12,00 & 4,00 \\
\hline \multirow{2}{*}{2012} & QM & 3 & 10 & 4 & 3 & 1 \\
\hline & $\%$ & 12,50 & 41,67 & 16,67 & 12,50 & 4,17 \\
\hline \multirow{2}{*}{2014} & QM & 5 & 8 & 4 & 4 & 1 \\
\hline & $\%$ & 21,74 & 34,78 & 17,39 & 17,39 & 4,35 \\
\hline \multirow{2}{*}{ Média } & $\mathrm{QM}$ & 3 & 10 & 5 & 3 & 0 \\
\hline & $\%$ & 11,54 & 38,46 & 19,23 & 11,54 & 0,00 \\
\hline
\end{tabular}

* QM: quantidade de municípios com Unidade de Conservação em Mato Grosso do Sul no período.

Não se observou, no período de 2002 a 2014, nenhum município com índice médio geral de eficiência igual a 1,0. Os que mais se aproximaram foram os municípios de Caracol, Ladário e Rochedo, com índices de $0,9986,0,9501$ e 0,9447 , respectivamente, muito próximos do índice de eficiência 1,0 (Tabelas 4 e 5).

Os municípios recebem os repasses, porém, os investimentos para contribuir com a implantação e a efetividade dos planos de manejo sucumbem à falta de uma gestão direcionada nas UCs (Tabelas 4 e 5).

A situação é ainda mais comprometedora na região da BH-RPR, por tratar-se de uma região com extensa exploração agroindustrial, gerando o comprometimento de um patrimônio extremamente importante para as atuais e futuras gerações por meio da degradação de mananciais de água e biodiversidade do ambiente, atentando desta maneira contra a preservação dos recursos naturais (Tabela 5).
Tabela 5 - Escores de eficiência municipal na Bacia Hidrográfica do Rio Paraná, quantidade de municípios e frequências percentuais desses municípios, de 2002 a 2014.

\begin{tabular}{|c|c|c|c|c|c|c|}
\hline $\begin{array}{l}\text { Escores de } \\
\text { eficiência }\end{array}$ & & $\begin{array}{l}0,1 \text { a } \\
<0,3\end{array}$ & $\begin{array}{l}0,3 \text { a } \\
<0,5\end{array}$ & $\begin{array}{l}0,5 \text { a } \\
<0,8\end{array}$ & $\begin{array}{c}0,8 \text { a } \\
<1,0\end{array}$ & 1 \\
\hline \multirow{2}{*}{2002} & QM & 11 & 3 & 3 & 7 & 1 \\
\hline & $\%$ & 44,00 & 12,00 & 12,00 & 28,00 & 4,00 \\
\hline \multirow{2}{*}{2004} & QM & 5 & 10 & 6 & 7 & 1 \\
\hline & $\%$ & 17,24 & 34,48 & 20,69 & 24,14 & 3,45 \\
\hline \multirow{2}{*}{2006} & $\mathrm{QM}$ & 8 & 5 & 10 & 12 & 1 \\
\hline & $\%$ & 22,22 & 13,89 & 27,78 & 33,33 & 2,78 \\
\hline \multirow{2}{*}{2008} & QM & 11 & 3 & 9 & 10 & 1 \\
\hline & $\%$ & 32,35 & 8,82 & 26,47 & 29,41 & 2,94 \\
\hline \multirow{2}{*}{2010} & QM & 9 & 2 & 12 & 17 & 1 \\
\hline & $\%$ & 21,95 & 4,88 & 29,27 & 41,46 & 2,44 \\
\hline \multirow{2}{*}{2012} & QM & 9 & 3 & 16 & 13 & 1 \\
\hline & $\%$ & 21,43 & 7,14 & 38,10 & 30,95 & 2,38 \\
\hline \multirow{2}{*}{2014} & QM & 2 & 8 & 21 & 16 & 1 \\
\hline & $\%$ & 4,17 & 16,67 & 43,75 & 33,33 & 2,08 \\
\hline \multirow{2}{*}{ Média } & QM & 8 & 6 & 21 & 15 & 0 \\
\hline & $\%$ & 16,00 & 12,00 & 42,00 & 30,00 & 0,00 \\
\hline
\end{tabular}

* QM: quantidade de municípios com UC em MS no período.

Maganhotto et al. (2014) discutem os aspectos de deficiências crônicas da falta de sustentabilidade das UCs no Brasil em razão de problemas como a inexistência e a desatualização dos Planos de Manejo e a carência de metodologias de suporte ao Zoneamento Ambiental, aspectos que reforçam a necessidade de novos estudos que identifiquem critérios e parâmetros aplicáveis ao planejamento ambiental em busca da sustentabilidade em UCs.

Outros aspectos que têm comprometido a sustentabilidade de UCs e do ambiente são a explosão demográfica, a crescente industrialização, a mecanização agrícola e os sistemas de monocultura, assim como a generalizada implantação de paisagens construídas e a intensa exploração dos recursos energéticos e de matérias-primas, que têm alterado, por vezes de modo irreversível, a natureza e comprometendo aspectos importantes da conservação dos recursos naturais (Favero et al., 2007). 
Ressalta-se que, na acepção proposta para o estudo, de que a eficiência é relativa e os insumos (inputs) e o produto (outputs), utilizados na análise, proporcionem um diagnóstico da relação PSA versus hectares de áreas das UCs, bem como das áreas dos municípios, onde se considera que índices de eficiências inferiores a 0,80 representam níveis de desenvolvimento insustentáveis em relação às UCs, não se obteve nos municípios das duas bacias ambientais estudadas nenhum caso de média eficiente (índice 1,0), o que indica que as políticas públicas podem avançar muito no sentido de tornar mais efetiva a preservação do ambiente.

Em estudos como os de Prado Filho \& Sobreira (2007), Ring (2008), Lopes \& Dias (2013), Euclydes (2013) e Ayach et al. (2014), o ICMS Ecológico é abordado como gênese de uma aproximação das políticas e processos públicos e privados de formação e incentivo à criação de UCs, embora nestes estudos não se construa uma análise da situação das unidades e sua representatividade.

Em alguns estudos, verificou-se a importância de desenvolver conhecimentos sobre as UCs e distinguir a abrangência dos resultados da preservação do ambiente, o que permite adicionar valor à conservação dos recursos naturais, seja em discussão dos níveis de conservação e das consequências para a saúde pública e tratamento de resíduos sólidos, como citado por Prado Filho \& Sobreira (2007) e Favaro \& Rossin (2014), seja pela análise de consequências do PSA e das UCs em relação ao adequado uso e ocupação da terra (Lopes \& Dias, 2013; Ayach et al., 2014).

Existem ainda estudos que se pautam pela convergência de mecanismos financeiros para a preservação do ambiente, como Silva (2013), Silveira et al. (2013) e Ribeiro et al. (2013), todos compactuando com a discussão proposta neste trabalho de identificar, demonstrar e avaliar os potenciais para desenvolvimento de conservação do ambiente, partindo de um princípio comum de relacionar o PSA proposto pelo ICMS Ecológico e políticas públicas adequadas de conservação.

Como preconizam Antoniazzi \& Shirota (2007) e Prado Filho \& Sobreira (2007), ao condicionar o repasse do recurso do ICMS Ecológico ao acompanhamento do desenvolvimento e conservação da UC por parte da esfera estadual, muda-se a esfera de decisão em prol de melhorias em UC, por exigir políticas efetivas de preservação do ambiente.

Desta forma, poderiam existir as condições para que as UCs tenham a correlata atenção pública em termos de investimento, acompanhamento e mitigação, para que tanto a sociedade quanto proprietários rurais e a gestão municipal possam gozar os benefícios de preservar o ambiente e os recursos naturais dele advindos.

Apesar de o principal motivo de criação das UCs ser a conservação da natureza, o poder público municipal é o principal incentivador à criação dessas unidades de conservação, visando aos benefícios financeiros gerados pelo ICMS Ecológico. Também se destaca a questão de que a maior parte das UCs é ainda recente e não apresenta qualquer tipo de planejamento nem plano de manejo, por causa da falta de apoio técnico, por ser um documento oneroso ou simplesmente por desconhecer a necessidade e a importância de tê-lo.

O que foi observado é que, apesar dos repasses vultosos para alguns municípios, os objetivos do ICMS ecológico ainda não foram atingidos, pois grande parte das unidades de conservação do estudo possui baixa eficiência ecológica.

\section{Conclus̃̃es}

Após 13 anos de implantação do ICMS Ecológico no Estado do Mato Grosso do Sul, a área em hectares das Unidades de Conservação, em relação à área total dos municípios, cresceu consideravelmente, ficando claro que os municípios estão buscando se adequar à legislação, aproveitando os seus benefícios.

Os valores repassados também sofreram um aumento considerável, tornando-se, em alguns casos, uma importante fonte de renda para municípios de pequeno porte, embora seja flagrante que a reciprocidade em relação à manutenção proativa de iniciativas de preservação, a partir do uso dos recursos dos PSA do ICMS Ecológico, não é acompanhada de um comportamento construtivo adequado.

Contrapondo-se aos aspectos observados de evolução na constituição de UCs, verifica-se que na maior parte desses municípios as suas UCs encontram-se aquém dos níveis de eficiências de manejos e conservação 
almejados para preservação do ambiente, ou seja, a maior parte dos municípios não mantém instituída uma política adequada de reciprocidade do PSA do ICMS Ecológico em ações para manutenção das unidades de conservação, tornando difícil a essas UCs manter níveis satisfatórios crescentes de conservação dos recursos naturais.

Neste estudo se pôde concluir a insuficiência na adoção de políticas de conservação, com os índices médios de eficiências obtidos pelos municípios na BH-RPY, destacando-se índices de municípios como Alcinópolis $(0,4018)$, Aquidauana $(0,4708)$, Bodoquena $(0,4261)$, Bonito $(0,3865)$, Corumbá $(0,3534)$, Coxim $(0,6992)$, Jardim (0,3701), Miranda $(0,4686)$ e Nioaque $(0,4740)$, importantes representantes do ambiente pantaneiro que mostram situação em que o ambiente e sua biodiversidade não estão tendo a devida atenção para sua conservação e preservação.

A mesma conclusão foi alcançada com os índices de eficiências obtidos BH-RPR, também com comportamento deficitário, com destaque para os municípios de Água Clara (0,4073), Brasilândia (0,2929), Campo Grande $(0,7067)$, Douradina $(0,1459)$, Dourados $(0,2506)$, Jatei $(0,2960)$, Maracajú $(0,2042)$, Naviraí $(0,5207)$ e Três Lagoas $(0,1334)$, demonstrando falta da devida reciprocidade de conservação do ambiente

\section{Referências}

Angulo-Meza, L.; Lins, M. P. E. Review of methods for increasing discrimination in Data Envelopment Analysis. Annals of Operations Research, 116, 225-242, 2002.

Angulo-Meza, L.; Biondi Neto, L.; Soares de Mello, J. C. C. B.; Gomes, E. G. ISYDS - Integrated System for Decision Support (SIAD - Sistema Integrado de Apoio à Decisão): A software package for Data Envelopment Analysis Model. Pesquisa Operacional, 25(3), 493-503, 2005.

Angulo-Meza, L., Biondi Neto, L., Soares de Mello, J. C. C. B.; Gomes, E. G.; Fernandes, A. J. S. Seleção de variáveis em DEA aplicada a uma análise do mercado de energia eléctrica. Investigação Operacional, 27, 21-36, 2007.

Antoniazzi, L.; Shirota, R. Pagamentos por serviços ambientais da agricultura para proteção de bacias hidrográficas. Anais e o iminente risco de perda de qualidade ambiental nas áreas protegidas.

Observa-se, como resultado do estudo, que há um comportamento incipiente das iniciativas de PSA em MS em atender a demanda por fomento financeiro e investimento na preservação dos recursos naturais.

$\mathrm{O}$ adequado direcionamento de recursos para financiar a efetivação de planos de manejo para proteção das UCs não vem sendo atingido. Como consequência, a falta de funcionários e de aparelhamento para a fiscalização contribui para o declínio dos aspectos de conservação em MS, embora existam iniciativas localizadas em áreas públicas e também em áreas privadas, como em RPPN's, onde os proprietários vêm priorizando a contratação de funcionários e a captação de recursos para conservação do ambiente.

\section{Agradecimentos}

Ao IMASUL - Instituto de Meio Ambiente de Mato Grosso do Sul -, gerência de Unidades de Conservação, pelas informações e pelo apoio para a realização do estudo; à CAPES (Coordenação de Aperfeiçoamento de Pessoal de Nível Superior) pela bolsa de estudos concedida. do XLV Congresso da Sociedade Brasileira de Economia, Administração e Sociologia Rural-SOBER, p. 134-156, 2007.

Ayach, L. R.; Bacani, V. M.; Da Silva, J. F. Unidades de Conservação no Pantanal do município de Aquidauana-MS: uma análise da evolução do uso da terra e cobertura vegetal e suas implicações. Cadernos de Geografia, 24(2), 138-154, 2014.

Banker, R. D.; Charnes, A.; Cooper, W. W. Some models for estimating technical scale inefficiencies in Data Envelopment Analysis. Management Science, 30(9), 1078-1092, 1984.

Bettencourt, L. M. A.; Kaur, J. Evolution and structure of sustainability science. PNAS, 108(49), 19540-19545, 2011.

Charnes, A.; Cooper, W. W.; Rhodes, E. Measuring the efficiency of decision-making units. European Journal of Operational Research, 2, 429-444, 1978. 
Euclydes, A. C. P. Contradições da política ambiental por meio de incentivos financeiros: os casos do ICMS Ecológico e da CFEM nos municípios do Quadrilátero Ferrífero (Minas Gerais, Brasil). Revista Árvore, Viçosa, 37(6), 1083-1092, 2013.

Farley, J.; Aquino, A.; Daniels, A.; Moulaert, A., Lee, D.; Krause, A. Global mechanisms for sustaining and enhancing PES schemes. Ecological Economics, 69, 2075-2084, 2010.

Favaro, A. K. M. I.; Rossin, A. C. Payments for environmental services that contribute to environmental health, a local level analysis. Saúde e Sociedade, 23(1), 216-226, 2014.

Favero, O. A.; Nucci, J. C.; De Biasi, M. Unidades de paisagem e zoneamento ambiental: subsídios para a gestão da floresta nacional de Ipanema - Iperó/SP. RA'E GA, 14, 35-53, 2007.

Frey, K. Políticas públicas: um debate conceitual e reflexões referentes à prática da análise de políticas públicas no Brasil. Planejamento e Políticas Públicas, 21, 211-259, 2000.

Kemkes, R.; Farley, J.; Koliba, C. J. Determining when payments are an effective policy approach to ecosystem service provision. Ecological Economics, 69, 2069-2074, 2010.

Kosmus, M.; Cordero, D. Payments for environmental services: An instrument to maintain global ecosystems. The International Journal for Rural Development, 43(1), 12-17, 2009.

Kosoy, N.; Corbera, E.; Brown, K. Participation in payments for ecosystem services: case studies from the Lacandon rainforest, Mexico. Geoforum, 39, 2073-2083, 2008.

Lopes, J. B. S.; Dias, R. R. Verificação do uso de SIG na implementação de sistema de Pagamento por Serviços Ambientais (PSA) em Bacias Hidrográficas do Estado do Tocantins. Ciência Equatorial, 3(1), 1-8, 2013.

Loureiro, W. Contribuição do ICMS Ecológico à conservação da biodiversidade no Estado do Paraná. 189f. Curitiba, Tese (Doutorado em Engenharia Florestal) - Programa de Pós-Graduação em Engenharia Florestal da Universidade Federal do Paraná, Curitiba, 2002.

Lustosa, M. C. J.; Young, C. E. F. Política ambiental. In: Kupfer, D.; Hasenclever, L. (Orgs.). Economia industrial: fundamentos teóricos e práticos no Brasil. Rio de Janeiro: Campus, 2002. 640p.

Lustosa, M. C. J.; Cánepa, E. M.; Young, C. E. F. Política Ambiental. In: May, P. H.; Lustosa, M. C.; Vinha, V. Economia do Meio Ambiente: teoria e prática. 2. ed. Rio de Janeiro: Campus Elsevier, 2003. 600p.

Maganhotto, R. F.; Santos, L. J. C.; Nucci, J. C.; Lohmann, M.; Souza, L. C. D. P. Unidades de Conservação: limitações e contribuições para a conservação da natureza. Sustentabilidade em Debate, 5(3), 203-221, 2014.

Maler, K. G.; Aniyar, S.; Jansson, A. Accounting for ecosystem services as a way to understand the requirements for sustainable development. Gretchen A Daily, 105(28), 9501-9506, 2008.

Mato Grosso do Sul. Decreto n. 10.478, de 31 de agosto de 2001. Estabelece métodos para o rateio da parcela de receita de ICMS pertencente aos municípios... Campo Grande, 2001.

May, P. H.; Lustosa, M. C.; Vinha, V. (Org.). Economia do Meio Ambiente: teoria e prática. 2. ed. Rio de Janeiro: Campus Elsevier, 2003. 600p.

Monte, M. A.; Silva, M. L. Análise do repasse do ICMS Ecológico aos municípios do Estado de Minas Gerais. Cerne, 15(4), 391-397, 2009.

Nascimento, V. M.; Nascimento, M.; Van Bellen, H. M. Instrumentos de políticas públicas e seus impactos para a sustentabilidade. Gestão \& Regionalidade, 29(86), 77-87, 2013.

Neves, E. M. S. C. Política ambiental, municípios e cooperação intergovernamental no Brasil. Estudos Avançados, 26(74), 137-150, 2012.

Nohara, J. J.; Souza, R. S.; Pinto, V. P. S.; Pinto, J. S. ICMS Ecológico como fator incentivador do desenvolvimento sustentável: preservação das bacias hidrográficas. Revista de Gestão Social e Ambiental - RGSA, 5(3), 102-115, 2011.

O'Farrell, P. J.; De Lange, W. J.; Le Maitre, D. C.; Reyers, B.; Blignaut, J. N.; Milton, S. J.; Atkinson, D.; Egoh, B.; Maherry, A.; Colvin, C.; Cowling, R. M. The possibilities and pitfalls presented by a pragmatic approach to ecosystem valuation in an arid biodiversity hotspot. Journal of Arid Environments, 75, 612-623, 2011.

Pagiola, S.; Arcenas, A.; Platais, G. Can payments for environmental services help reduce poverty?: an exploration of the issues and the evidence to date from Latin America. World Development, 33(2), 237-253, 2005.

Petheram, L.; Campbell, B. M. Listening to locals on payment for environmental services. Journal of Environmental Management, 91, 1139-1149, 2010.

Prado Filho, J. F.; Sobreira, F. G. Operational and environmental performance of sanitary landfills and recycling facilities supported by the ecological ICMS in Minas Gerais State, Brazil. Revista Engenharia Sanitária e Ambiental, 12(1), 52-61, 2007.

Ribeiro, C. D. A.; Rossato, M. V.; Guse, J. C.; Freitas, L. A. R.; Dorr, A. C. ICMS Ecológico: uma abordagem à gestão am- 
biental nos municípios do Rio Grande do Sul. Revista Sociais e Humanas, 26(2), 363-383, 2013.

Ring, I. Integrating local ecological services into intergovernmental fiscal transfers: the case of the ecological ICMS in Brazil. Science Direct Land Use Policy, 25, 485-497, 2008.

Rossi, A.; Martinez, A. L.; Nossa, V. ICMS Ecológico sob o enfoque da tributação verde como meio da sustentabilidade econômica e ecológica: experiência do Paraná. Revista de Gestão Social e Ambiental - RGSA, 5(3), 90-101, 2011.

Santos, R.; Ring, I.; Antunes, P.; Clemente, P. Fiscal transfers for biodiversity conservation: the Portuguese local finance Law. Land Use Policy, 29, 261-273, 2012.

Senra, L. F. A. C.; Nanci, L. C.; Soares de Mello, J. C. C. B.; Angulo-Meza, L. Estudo sobre métodos de seleção de variáveis em DEA. Pesquisa Operacional, 27(2), 191-207, 2007.
Silva, J. I. A. O. Conservação de recursos naturais no semiárido e desenvolvimento: análise do caso das reservas privada. Ambiente \& Sociedade, 16(1), 79-88, 2013.

Silveira, V. C.; Cirino, J. F.; Prado Filho, J. F. Economic valuation of the state environmental protection area of Cachoeira das Andorinhas-MG. Revista Árvore, 37(2), 257-266, 2013.

Soares de Mello, J. C. C. B.; Gomes, E. G.; Angulo-Meza, L.; Biondi Neto, L.; Sant'Anna, A. P. Fronteiras DEA difusas. Investigação Operacional, 25(1), 85-103, 2005.

Soares de Mello, J. C. C. B.; Angulo-Meza, L.; Gomes, E.G.; Biondi Neto, L. Evaluación de la concentración en una ruta aérea brasilera con modelo DEA y frontera invertida. Revista de la Facultada de Ingeniería, 14(1), 64-71, 2006.

The Nature Conservancy. ICMS Ecológico, 2014. Disponível em: $<$ http://www.icmsecologico.org.br/>. 